\title{
¿Televisión de proximidad o televisión local? El impacto de la proximidad cultural en los estudios de televisión en la era digital
}

\author{
Gertutasun kulturalak telebistaren inguruko azterketatan duen eragina \\ Local television or proximity television? The impact of cultural \\ proximity on television studies in the digital era
}

\author{
Aida Martori` \\ Universidad Autónoma de Barcelona
}

\begin{abstract}
RESUMEN: El término televisión local ha sido tradicionalmente utilizado para hacer referencia a los prestadores que informan sobre lo que sucede en un municipio o ciudad. Sin embargo, este artículo pretende abrir un diálogo acerca del término televisión de proximidad que, en base a las teorías de proximidad cultural, se considera un concepto más amplio y preciso para referirse a un fenómeno cada vez más heterogéneo. Así, permite aludir a una realidad diversa de prestadores que van desde medios hiperlocales, que puede abarcar desde televisiones de un barrio de una ciudad, hasta televisiones supramunicipales o incluso regionales.
\end{abstract}

PALABRAS CLAVE: Televisión de proximidad; televisión local; proximidad cultural, representación; identidad local.

ABSTRACT: The term local television has traditionally been used to refer to providers who report on what happens in a municipality or city. However, this article aims to open a dialogue about the term proximity television that, based on theories of cultural proximity, is considered a broader and more precise concept to refer to an increasingly heterogeneous phenomenon. Thus, it allows to refer to a diverse reality of providers ranging from hyperlocal media, which can be televisions in a neighborhood of a city, to supramunicipal or even regional televisions.

KEYWORDS: Proximity television; local television; cultural proximity; representation; local identity.

\footnotetext{
* Correspondencia a / Corresponding author: Aida Martori. Facultat de Ciències de la Comunicació, UAB, Plaça Cívica (08193 BellaterraBarcelona) - aida.martori@uab.cat - https://orcid.org/0000-0003-0363-1636

Cómo citar / How to cite: Martori, Marisol; Aida (2019). "¿Televisión de proximidad o televisión local? El impacto de la proximidad cultural en los estudios de televisión en la era digital», Zer, 25(47), 147-163. (https://doi.org/10.1387/zer.21073).

Recibido: 20 agosto, 2019; aceptado: 26 septiembre, 2019.

ISSN 1137-1102 - eISSN 1989-631X / (c) 2019 UPV/EHU

(c) (i) Esta obra está bajo una licencia

Creative Commons Atribución 4.0 Internacional
} 


\section{Introducción y metodología}

En los últimos años diversos autores han prestado atención al fenómeno de la televisión local y regional (Corominas, et al., 2007; Corominas, 2009; Costera, 2010; Domingo, Alborch y Araüna, 2009; Fernández Alonso, et al., 2007; Guimerà, 2006a; Guimerà, 2007; Guimerà, Monedero y Martori, 2016; Jankowski y Prehn, 2002; López, 2000; Martori, 2016; Martori, 2014; Monedero: 2012; Prado y Moragas, 2002; Williams, Barnett, Harte, y Townend, 2014). A pesar del interés por la temática y la existencia de numerosos estudios de caso, se ha detectado una insuficiente conceptualización teórica orientada a definir los términos utilizados para hacer referencia a estos prestadores, así como una falta de debate académico en este sentido. Para el análisis en profundidad de varios casos y para contribuir al debate internacional sobre esta área de investigación, se ha creído determinante desarrollar un estudio sobre el concepto de televisión de proximidad. Se considera el término más preciso para hacer referencia a una realidad diversa y heterogénea, pero con unos rasgos comunes.

En el marco de globalización mediática aumenta la demanda de los ciudadanos de recibir información de las áreas más próximas a su identidad, donde los ciudadanos desarrollan la mayor parte de sus vidas. Es aquí donde radica la importancia de los prestadores de proximidad, para mantener y reproducir los rasgos identitarios y culturales y evitar, así, que se diluyan ante los flujos globales. Un acercamiento teórico al concepto es relevante, especialmente en un momento en que varios autores hacen una mirada a los prestadores locales y regionales, que son una alternativa ante las lógicas globalizadoras de la comunicación y los grandes players que tienen impacto en todo el mundo.

Los medios de comunicación locales han sido un objeto de estudio ampliamente investigado en la segunda mitad del siglo xx. El concepto local contiene limitaciones que hay que superar, sobre todo en cuanto a la diversidad de realidades a las que se refiere, que no siempre coinciden con los límites territoriales de una localidad. El término proximidad es útil para ir más allá de la denominación histórica de comunicación local, comarcal o regional.

Este artículo se basa en una metodología cualitativa, consistente en la consulta bibliográfica y de documentos oficiales e inéditos, además de entrevistas a expertos y profesionales realizadas para analizar el estudio de caso. Para conceptualizar la televisión de proximidad, se repasará el contexto y ámbitos en los que el término ha sido utilizado, y se tendrá en cuenta una visión internacional, partiendo de las teorías de la proximidad cultural. Se actualizará el concepto al máximo para enmarcarlo en la era digital, y se aplicará al estudio de caso de los prestadores de proximidad en Cataluña con el objetivo de demostrar la aplicabilidad del análisis teórico. 


\section{Estado de la cuestión y contexto}

Un antecedente a la interpretación del concepto proximidad hace referencia a la mesocomunicación. Miquel de Moragas definió cuatro ámbitos de comunicación: megacomunicación, macrocomunicación, mesocomunicación y microcomunicación (Moragas, 1985). Dentro de esta catalogación, la mesocomunicación se situaba entre la gran comunicación y las pequeñas circulaciones informativas en el ámbito interpersonal y grupal, con una fuerte relación entre los contenidos que se emiten y el activismo social.

A pesar de este intento de teorizar el objeto de estudio y de dar más visión y notoriedad a la comunicación local, el concepto no llegó a sustituir la denominación de comunicación local y entró en desuso en los años noventa (Guimerà, 2012). En esta década, el término local seguía siendo el más utilizado para denominar los medios de comunicación que ofrecían una información cercana al territorio. Sin embargo, este artículo expone por qué, dadas las particularidades de los prestadores a la era digital, el término proximidad pasa a ser el más adecuado para referirnos a unos medios que ofrecen contenido referido a la capa identitaria más próxima a los individuos.

La aplicación del concepto proximidad a los medios apareció en el ámbito de la televisión. Fue utilizado por primera vez en Francia, a finales de los años ochenta, como télévision de proximité (Guimerà, 2012). Es de especial relevancia en esta investigación destacar que la primera referencia pretendía denominar las desconexiones que había empezado a hacer el canal público de cobertura nacional France 3. Es notorio el hecho de que el término en primera instancia no apareciera en referencia a las televisiones de cobertura local y supralocal, sino que se pretendía dar respuesta al tipo de información próxima al territorio que emitía una televisión de alcance estatal mediante la fórmula de la desconexión, de manera que cada territorio recibía las informaciones referidas al ámbito geográfico que le era propio.

Los investigadores belgas Pierre Fastrez y Stéphanie Meyer desarrollaron el concepto de proximidad a finales de los años 90. Consideraban que la relación de proximidad entre televisión local y receptor se establecía a través de la programación, basada en tres relaciones:

- Medio de comunicación y receptor.

- Medio de comunicación y mundo referencial.

- Receptor y mundo referencial.

Esta interpretación del concepto está en consonancia con el hecho de dar importancia a los contenidos, al considerar que estos son cercanos si se refieren al terri- 
torio concreto en el que emiten, y que los contenidos cercanos en un determinado lugar no lo serán en otro.

El grupo de investigación EURORETV volvió a usar este término, en la búsqueda de una nomenclatura que delimitara un objeto de estudio que comprendía una gran diversidad de modelos televisivos (Guimerà, 2012). Miquel de Moragas, Carmelo Garitaonandia y Bernat López se referían a las experiencias de emisión a «pequeña y mediana escala», en una investigación sobre las televisiones de proximidad en Europa en 1999. En este sentido, se seguía con la línea iniciada en Francia de prestar atención a los contenidos informativos relativos a un territorio, a la hora de definir el término.

El concepto de proximidad aplicado a la televisión tiene que ver con la idea que entre la emisora y sus receptores existe un escenario de experiencias compartidas, cosa que, en definitiva, se verá reflejada en los contenidos de la programación. (Moragas, Garitaonandía i López, 1999: 19)

La televisión de proximidad también fue definida por Prado (2004) como aquella que se dirige a una comunidad local delimitada territorialmente, y que lo hace con contenidos relativos a la experiencia cotidiana de los receptores, a sus preocupaciones, a su patrimonio cultural ya su memoria histórica, con la voluntad de incrementar la participación social de la comunidad. «Proximidad hace referencia al área geográfica de difusión (local), pero sobre todo se refiere a los usos sociales de los contenidos» (Prado, 2004: 32).

En este contexto, el concepto proximidad implica el reconocimiento de un escenario compartido entre emisor y audiencia, que queda reflejado en el contenido final de la programación, especialmente los telediarios (Prado y Moragas, 2002). Los autores defendían que cuando se preservan estas misiones se pueden generar sinergias positivas con el conjunto del sistema audiovisual y de comunicación.

\begin{tabular}{|c|c|}
\hline Entorno general & Entorno general \\
$\uparrow$ atrae & $\downarrow_{\text {afecta }}$ \\
Información de proximidad & Información de proximidad \\
\hline
\end{tabular}

Fuente: elaboración propia.

Figura 1

\section{Cuadro de las dos vertientes de la información local}

En el intento de teorizar el objeto de estudio también es útil la aportación de Domingo, Alborch y Araüna (2009), que definen la información de proximidad 
como aquella que refleja acontecimientos y tendencias que se desarrollan en el entorno más inmediato de la audiencia. Esta definición se refiere al espacio donde tiene lugar la noticia y la relación de este espacio con el espectador.

Los autores distinguen entre las noticias de proximidad que pueden resultar atractivas en informativos generalistas, y las noticias de ámbito internacional, estatal o autonómico que pueden tener una vertiente más próxima, cuando se las da un punto de vista local y se interpretan de acuerdo con su afectación a los ciudadanos o las instituciones locales. El cuadro (figura 1) muestra las dos vertientes de la información local, en relaciones al contexto generalista.

Continuando con la definición del concepto proximidad de los investigadores Domingo, Alborch y Araüna (2009), se observa que desglosan el concepto de proximidad según:

- La repercusión de la noticia, en función de los ciudadanos a los que afecta, y de si puede ser útil para tomar decisiones y estar al día de lo que sucede en su entorno más inmediato.

- El ámbito geográfico, que se determina según los protagonistas y el escenario de una noticia.

— La visibilidad del mundo local, en función de si se han incluido fuentes locales, independientemente de la repercusión y el alcance geográfico de una información. Esta dimensión permite conectar las tendencias locales y la realidad próxima, que los ciudadanos entiendan las repercusiones de las informaciones de alcance local, y que comprendan su entorno inmediato. Esta dimensión corresponde a la segunda vertiente de la información local, en la que el ámbito local se puede presentar como origen o causa del hecho noticiable, o como un caso o ejemplo de la información, tanto si tiene repercusión local como general.

La principal concreción de la televisión de proximidad, según los autores, es la atención preferente de los prestadores a los contenidos informativos relativos al territorio de referencia.

Las aportaciones sobre televisión de proximidad nos permiten tener un punto de partida a la hora de analizar el concepto, centrado principalmente en el tipo de contenidos. A partir del repaso bibliográfico, este artículo pretende ofrecer una interpretación del fenómeno a partir de las teorías de la proximidad cultural. 


\section{Una aproximación a la proximidad cultural}

Joseph Straubhaar desarrolló la teoría de la proximidad cultural a principios de los años 90, y la reinterpretó a lo largo de los años 2000. Sus postulados ayudan a definir el concepto y dan pie a relacionar la proximidad cultural con los medios que informan de lo que sucede en el ámbito geográfico más próximos a la ciudadanía. Straubhaar ha aplicado la teoría de la proximidad cultural en el campo televisivo, e incluso al servicio público de radiodifusión, por lo que sus aportaciones han sido de utilidad en los estudios de televisión. La teorización de la proximidad cultural permite enmarcar el fenómeno de la televisión de proximidad en un espacio teórico estrechamente relacionado con una realidad de prestadores heterogénea, como se desarrollará en su aplicabilidad al estudio de caso.

La proximidad cultural está relacionada con la proximidad geográfica. La primera se entiende como los elementos de la cultura que se dan en un lugar determinado; y la segunda, como el espacio concreto en que las comunidades se relacionan. En los análisis del autor se relacionan estos dos conceptos en varias ocasiones, especialmente cuando se refiere a la identidad multicapa y cuando utiliza el término geografia cultural. La proximidad cultural y la geográfica están vinculadas y se retroalimentan, dado que el conjunto de elementos culturales configura la identidad y la identificación geográfica de los individuos, que se refleja en los medios de comunicación.

\subsection{El Papel aCtivo De la Audiencia}

Los medios de comunicación forman parte de una superestructura cultural que resulta de las relaciones económicas, en el que los productores locales coexisten con grandes producciones, principalmente norteamericanas y europeas. En el análisis de la estructura y el rol de los medios de comunicación de masas, en los años 70 y 80 se identifican flujos de un solo sentido, desde los países del primer mundo hacia el resto del mundo (Boyd-Barrett, 1980) en el campo de la televisión y de las agencias de noticias. La dependencia cultural expone que los medios forman parte de la estructura cultural que resulta de las relaciones económicas de dependencia.

Straubhaar (1991) hace una crítica al determinismo de este punto de vista por la supuesta limitación a razones económicas, dejando de lado el rol que juega la audiencia y sus interacciones. En este sentido, el autor introduce el papel de la audiencia como elemento clave para entender el sistema cultural, mediático y televisivo, y como actor determinante en la búsqueda de proximidad cultural en los medios de comunicación. Por tanto, en un primer momento incorporaba la audiencia en el debate sobre el imperialismo en los medios, como elemento activo en la búsqueda de proximidad en los bienes culturales. 
La dependencia evoluciona hacia una interdependencia asimétrica en el mercado de la televisión mundial, con una fuerte presencia del componente regional y local. En una esfera global, los individuos siguen buscando la proximidad cultural en los medios de comunicación, de manera que los contenidos globales conviven con aquellos más cercanos a los ciudadanos.

El público tiene un papel activo en la elección e interpretación de la oferta televisiva, y no es homogéneo. Fiske (1987) ya había puesto de manifiesto el papel activo de la audiencia en relación al medio televisivo, argumentando que las audiencias consumen televisión para obtener una comprensión de la realidad que los conecte con su experiencia social. Relacionaba, por tanto, espectadores con interacciones sociales, un postulado aplicable al caso de la televisión local, en el que el medio proyecta lo que pasa en la sociedad y retroalimenta la participación social.

Como centro de la aportación de Straubhaar (2008) hay que mencionar la teoría de que la audiencia busca la proximidad cultural, que significa que los ciudadanos tienen una preferencia por las producciones locales y nacionales, debido a diferentes factores: la atracción los rostros con rasgos étnicos similares, la familiaridad con los estilos locales, la atracción por las temáticas locales, el conocimiento local, la comprensión del sentido del humor o el reconocimiento de las personalidades locales.

\subsection{Las Múltiples Capas de IDENTIFICACIÓN}

Los individuos se identifican con una variedad de niveles (múltiples capas de identidad). En un contexto de globalización y de globalización mediática (Stiglitz, 2002; Mattelart, 1998), la identidad de los individuos no únicamente se globaliza o deviene transnacional, sino que conviven diferentes capas de identidad según la referencia geográfica (local, regional o transnacional) que se relacionan en base a ámbitos lingüísticos y nacionales (Anderson, 1983).

Estos niveles de identidad son articulados a través de los medios, en la que tienen un papel destacado la televisión e Internet. Los medios de comunicación, por lo tanto, se convierten en instituciones complejas y multicapa. Según Castells (1997), la identidad todavía tiende a basarse en el lenguaje, la religión, la geografia, la historia, el origen étnico, la memoria colectiva y los aparatos de poder político. Por lo tanto, los elementos de la identidad a menudo corresponden a niveles culturales más pequeños que la esfera global.

La televisión y las redes televisivas se globalizan, pero también se da un fenómeno paralelo de localización y regionalización, para mostrar historias específicas referentes a cada uno de los territorios y tradiciones culturales. Las demandas de adaptación a las realidades sociales más cercanas dan lugar a la localización de la televisión. 
Las industrias culturales tienen una tendencia a gestionar la interdependencia de las relaciones internacionales y una adaptación de formas importadas a nivel nacional. Emerge una situación de fuerte adaptación al nivel local de los patrones globales (Kraidy, 2005). Pero no solo eso, además de la importación de formatos y la adaptación a escala doméstica, los públicos buscan producciones que apelen a la realidad más próxima, y que provean informaciones propias referentes a la primera capa, la más cercana a los individuos.

Straubhaar (2007) menciona la presencia de una nueva capa llamada globalización, que no hace que desaparezcan las otras, más próximas al individuo. Las capas no están aisladas ni existen por separado, de manera que interactúan unas con otras, especialmente en el ámbito cultural. La proliferación de múltiples capas de identidad se relaciona con el uso de los medios. El autor diferencia los flujos de televisión, según diferentes tipologías que responden a las diversas capas.

Considera que existen las siguientes infraestructuras tecnológicas globales: un imperio norteamericano basado en Hollywood; otros exportadores globales (como es el caso de las telenovelas latinoamericanas o la animación japonesa); productores lingüístico-culturales transnacionales y productores geoculturales relacionados con la proximidad geográfica, las culturas, los lenguajes y las historias similares; productores translocales, que cruzan las fronteras (como es el caso de EEUU y México); productores nacionales; productores regionales; productores metropolitanos; y productores locales.

Según lo que llama geografía cultural, Straubhaar define la capa regional como la referida a la identidad y los medios que hacen referencia a un área más pequeña que el Estado, pero mayor que el estrictamente local. Como ejemplo, menciona regiones con una relevancia cultural y lingüística. Si se avanza hacia las capas más próximas, se puede destacar la diferenciación que hace el autor entre productores metropolitanos y productores locales. En el caso de los primeros, hace referencia a ciudades cosmopolitas que están relacionadas con las redes globales y que producen un elevado volumen de contenido propio, dirigido a la propia ciudad, pero también a las regiones, naciones o espacios transnacionales. Los productores locales se sitúan al nivel de ciudades más pequeñas, municipios o incluso barrios.

A partir de la concepción de la identidad como multicapa, se observa que hay una demanda por parte de los individuos de recibir información de las capas más amplias, es decir de la esfera global y transnacional, pero también de aquellas más cercanas los espacios donde los ciudadanos desarrollan su día a día: el Estado, la región e, incluso, la localidad. Es en las dos últimas capas en las que se sitúa el espacio para los prestadores de proximidad, como aquellos que informan de temáticas que tienen relación con la vida cotidiana de las personas. 
En el marco de la globalización y con Internet como fuente de consumo audiovisual cada vez más generalizado, se produce un fenómeno paralelo: los grandes flujos informativos de la superestructura cultural hacen que las fórmulas televisivas se globalicen cada vez más y, por otra parte, también crezca la necesidad de los ciudadanos de disponer de proyectos que den respuesta a sus necesidades de proximidad cultural. Las diferentes capas de identidad, reflejadas en los medios, no han hecho desaparecer la presencia de prestadores de televisión local ni han homogeneizado el mercado televisivo, haciendo patente la necesidad de los espectadores de disponer de proyectos comunicativos que reflejen los diferentes niveles de identidad.

\subsection{El MEdio teleVisivo EN RELACión a LAS Múltiples CAPAS DE IDENTIDAD}

Se produce una situación dicotómica, en el que la localización de los contenidos convive con la búsqueda por parte de los espectadores de propuestas que refuercen los aspectos de la identidad que hagan referencia a la nueva capa de identidad transnacional o global. Ante la multiplicación de la oferta comunicativa y televisiva, los prestadores locales juegan un papel destacado en la satisfacción de las demandas de los ciudadanos, respondiendo a la teoría de la proximidad cultural. La proximidad cultural explica la tendencia a preferir la producción local o nacional, y aquellos productos culturales que se encuentran cerca de la propia identidad de las personas. Las televisiones locales tienen la misión de ofrecer, también a través de Internet, aquellos productos audiovisuales más cercanos a la identidad local de los individuos.

En un contexto de globalización generalizada, las televisiones de proximidad representan la única oportunidad para los territorios, colectivos y ciudadanía de encontrar el reflejo en los medios de comunicación (Prado, 2004). Este hecho tiene una gran trascendencia como contrapeso a los procesos de globalización cultural, así como en términos de conservar la memoria histórica y prestar un servicio a la normalización lingüística en los territorios que tienen lenguas propias.

Teniendo en cuenta una investigación desarrollada en Holanda, Costera (2010) identifica siete funciones sociales que lleva a cabo la televisión local en relación a su audiencia: suministrar información de antecedentes (imparcial, fiable y amplia); fomentar la integración social y aportar conocimiento a los ciudadanos sobre cómo funciona la ciudad; garantizar la representación (dar voz y reconocimiento, y reflejar la sociedad); aumentar el conocimiento local; crear memoria cívica; contribuir a la cohesión social; y potenciar un sentido de pertenencia. Según la autora, las emisoras de televisión local constituyen una condición previa para la democracia, deben representar el área local y deben suministrar historias contrastadas sobre sus residentes. 
El mundo de los medios está fuertemente estratificado e Internet amplía el rango de capas de producción y de identificación, desde la esfera más global a la más local. Relacionando la geografía cultural con la tecnología, se puede afirmar que los proyectos online son más económicos y permiten la emergencia de nuevos portales de televisión y de noticias locales, que informen sobre lo que sucede en las ciudades, pueblos o barrios. De este modo aparecen nuevas fuentes de noticias con las que la audiencia se identifica, ya que son extremadamente localizadas.

En este sentido, hay que hablar de medios hiperlocales, un concepto que ya ha sido teorizado por algunos autores. Se trata de prestadores centrados en elementos geográficos, con orientación a la comunidad, que emiten información original, muchos de ellos nativos digitales, que llenan vacíos informativos percibidos y que están centrados en el compromiso cívico (Metzgar, Kurpius y Rowley, 2011). Su alcance se define por las necesidades de la comunidad a la que los prestadores hiperlocales van dirigidos $\mathrm{y}$, por tanto, puede ser variable, y su viabilidad viene dada por el potencial del entorno web que facilita su existencia.

Su misión es difícil de evaluar, pero está claramente relacionada con el deseo de desarrollo de herramientas adecuadas para la promoción de la gobernabilidad democrática, entendida como una oportunidad para los contenidos generados por el usuario o para activismo cívico y político. Williams, Barnett, Harte y Townend (2014) se refieren a los medios que cubren informaciones cívicas y de valor cultural, incluyendo noticias sobre la comunidad local, sus grupos, eventos y los asuntos de la gobernanza municipal.

Dentro de los medios que ofrecen información local, hay que hablar de un espacio heterogéneo en el que conviven los prestadores que informan sobre lo que sucede en un pueblo o ciudad, los que lo hacen para varios municipios con un ámbito de cobertura supralocal, y los que lo hacen en ámbitos sublocales o más pequeños que la localidad. La diversidad de prestadores no puede desligarse del aspecto tecnológico, ya que en muchos casos la naturaleza de los medios resulta de los condicionantes técnicos y de las políticas de comunicación. La transición de la televisión analógica a la digital ha supuesto en muchos países una reordenación de licencias que ha afectado a los prestadores de proximidad.

Si hablamos del caso español, por ejemplo, la escasez de licencias disponibles ha comportado que muchos de los prestadores locales se hayan convertido en supralocales y que varios municipios compartan un canal. En el Reino Unido también se observa que la cobertura de las licencias de TDT de proximidad supera en muchos casos el ámbito local. En Escocia, por ejemplo, los canales recientemente puestos en marcha en Aberdeen, Ayr y Dundee no informan solo sobre asuntos de estas ciudades, sino que también engloban el área de influencia de las mismas y se pueden ver en un territorio más amplio que el estrictamente local. 
En países en los que se están preparando para la digitalización, como pasa en América Latina, están planificando la distribución de licencias para que puedan operar televisiones de proximidad, ante el riesgo de que los grandes players las utilicen para emitir contenido generalista. En Costa Rica, ya son diversas las voces que han alertado del riesgo de no reservar espacio del espectro radioeléctrico para los prestadores de proximidad que cumplan la función de representar las áreas locales.

Paralelamente a las consecuencias del paso a la TDT, Internet también ha introducido cambios en el modelo de televisión de proximidad. Ha propiciado la aparición de numerosos medios locales que no han conseguido una licencia para operar, así como la aparición de webs de noticias que informan sobre lo que sucede en barrios o ámbitos más pequeños que un pueblo o ciudad, especialmente medios hiperlocales.

En un contexto en el que los espacios sociales relacionales se desterritorializan y se diluyen los rasgos identitarios, la televisión de proximidad tiene la posibilidad de ser el actor que justamente se encargue de afianzar de nuevo las identidades locales porque no se diluyan en la globalidad.

\section{Televisión de proximidad: el caso catalán}

Cataluña ha desarrollado un espacio comunicativo propio (Gifreu y Corominas, 1991). Zallo (2013) concreta una de las particularidades del espacio catalán de comunicación como la existencia de un eje nacionalista y la concepción de que hay una provisión dual de contenido en los medios: el producido dentro y el producido fuera de Cataluña.

En el caso catalán, la construcción identitaria está estrechamente vinculada al espacio de comunicación, y se relaciona de manera directa con el elemento lingüístico y la normalización del catalán. Dentro de un espacio comunicativo como Cataluña entrecruzan diferentes niveles, por encima y por debajo, en relación a las múltiples capas de identidades de sus habitantes, siguiendo los postulados de la identidad multicapa. Los prestadores de proximidad han sido herramientas dinamizadoras y transmisoras de la cultura popular. Estos medios, tradicionalmente, han tenido un ámbito de cobertura municipal y la comunidad investigadora se ha referido a ellos como televisiones locales.

Esta realidad ha evolucionado, especialmente desde el paso a la TDT, en el que la mayor parte de las televisiones pasaron a tener un ámbito de cobertura supramunicipal. Si bien en la década de los 90 y 2000, los prestadores de proximidad en Cataluña eran básicamente locales, con un ámbito de cobertura y difusión que coincidía con los límites de un municipio o ciudad, en los últimos años, se observa una tendencia a la diversificación de los prestadores de proximidad. 
El Plan Técnico Nacional de TDT preveía que pudiera haber 96 canales de televisión con ámbitos de difusión muy diversos estructurados en demarcaciones. Había demarcaciones de tres municipios y otras que agrupaban una treintena de poblaciones. Además, dentro de las demarcaciones podía haber adjudicaciones de licencias que correspondían a un solo ayuntamiento para emitir contenido relativo al pueblo o ciudad, a pesar de que el ámbito de difusión fuera toda la demarcación (es el caso de Badalona, Barcelona o l'Hospitalet de Llobregat). Por lo tanto, se rompía el vínculo de un municipio-un canal que existió durante la etapa analógica, según el cual los prestadores emitían en base a un ámbito de difusión y de cobertura local.

Con el paso a la TDT, se apostó por la gestión conjunta de los canales públicos por parte de diferentes municipios que querían disponer de un canal de proximidad. De esta manera, ninguna población perdía el derecho a disponer de un canal público, si bien en la mayor parte de los casos tenía que ser compartido. No obstante, a lo largo de los años algunos municipios han perdido el interés por hacer televisión y han salido de los consorcios, a lo que cabe sumar que algunos proyectos televisivos no se llegaron a impulsar.

No sólo la digitalización fue la causa de la modificación que sufrió el modelo, pero es cierto que la organización de la TDT-L mediante demarcaciones afectó la estructura de los prestadores. Otro factor a tener en cuenta son los elevados costes de distribución de la señal de esta tecnología, que hicieron que muchos de los canales que están emitiendo se concentren en el área metropolitana o en zonas pobladas. Hay que considerar también el fuerte impacto de la crisis económica en un sector económicamente precario, en un momento de redefinición del modelo en el que se requerían inversiones por parte de los municipios para impulsar o dar continuidad a los proyectos.

A resultas de la encedida digital (o el apagón analógico, según algunos autores), hay prestadores que están gestionados por más de un municipio y que, por tanto, son supramunicipales. Sin embargo, el área de cobertura y la de difusión no tienen por qué ser coincidentes, dado que hay municipios de una demarcación que no se han integrado en los proyectos. Por ejemplo, TAC12 se difunde por tres comarcas del Camp de Tarragona (que incluyen 67 municipios) pero sólo informa de lo que sucede en los municipios que forman parte del Consorcio, que son siete.

Hay otros canales que son unimuniciales y que emiten de manera irregular por el pueblo o ciudad al que dan cobertura, y otros que, a pesar de dar cobertura a un pueblo o ciudad, se emiten por un ámbito de cobertura mucho más amplio, como es el caso de Barcelona Televisión (betevé). Aparte, se han impulsado muchas iniciativas que emiten por Internet, con áreas de cobertura diversas, que pueden ser barrios, pueblos o áreas más amplias. Como ejemplo, se pueden mencionar varias televisiones de distrito de Barcelona, que emiten información referida a los barrios de la 
ciudad, o bien Televisión de Cardedeu, que no optó al concurso para gestionar un canal de TDT y pasó a emitir online.

A menudo estos medios de comunicación presentan unas características que dificultan la identificación y definición del ámbito de cobertura y el área de difusión, que, como se ha detallado, no tienen por qué ser coincidentes ni corresponder a una sola localidad. Por este motivo, el estudio de caso va en el mismo sentido que la argumentación teórica y terminológica de este artículo: se considera más preciso utilizar un concepto que permita llamar a estos medios por el modelo y tipo de información que emiten, más que por el territorio que engloban. Con la digitalización, el concepto de comunicación de proximidad responde mejor a los retos que plantea la multiplicidad de fórmulas comunicativas sublocales, locales y supramunicipales.

De hecho, cuando se planificó la transición a la TDT ya se previó que habría que redefinir el concepto de televisión local para situarlo en el ámbito de la proximidad. En una reunión de académicos e intelectuales, celebrada antes de la adjudicación de licencias y organizada por la Dirección General de Medios de Comunicación de la Generalitat antes del apagón analógico, surgió la siguiente definición:

Se entiende por televisión de proximidad la que está gestionada por una empresa o institución, pública o privada, con o sin ánimo de lucro, vinculada al territorio y con infraestructuras de producción en la demarcación de actividad, y que emite, predominantemente en catalán, contenidos informativos (veraces y plurales), de creación, de entretenimiento e interactivos, fundamentalmente relacionados con lo que sucede en el ámbito cercano, geográfico, humano, cultural, lingüístico e histórico, y que promueve mecanismos de acceso y de participación de los ciudadanos. (Reunión de expertos, 2004)

El término televisión de proximidad, profundamente definido en este texto a partir de las teorías de la proximidad cultural, sirve para aludir a una realidad diversa que permite dar respuesta a la naturaleza múltiple de los prestadores de televisión actual, en el marco de la era digital. Las televisiones de proximidad engloban las televisiones supralocales, locales, y las de barrio o distrito. Permite incluir, por tanto, el fenómeno de los prestadores hiperlocales. Así pues, el concepto de televisión de proximidad contempla los prestadores locales, pero también los que dan servicio a un conjunto de municipios o a áreas más pequeñas de un pueblo o ciudad.

Ante esta variedad de prestadores, es básico llamarlos en función del servicio que prestan y de su función principal. En este sentido, la diversidad de medios que se han descrito se regirían por la denominación de prestadores de proximidad siempre que tengan como misión principal la producción y emisión de informaciones del territorio más cercano, y referentes a las capas de identidad más próximas de los individuos. 


\section{Conclusiones}

Las particularidades de la era digital, en la que los prestadores emiten por TDT en áreas de cobertura diversas y también online, el concepto televisión de proximidad se adecua mejor a la realidad existente que el concepto televisión local, que era el mayoritariamente utilizado por la comunidad investigadora. Este artículo pone de manifiesto que el término proximidad es más preciso y permite aludir a una realidad mucho más heterogénea de prestadores. Se trata de una adaptación terminológica pero también conceptual, cuidadosamente descrita y justificada, que da respuesta a los cambios que se han producido en el sector audiovisual de proximidad.

En este sentido, se apuesta por poner el foco en la función de los prestadores en relación a la satisfacción de las necesidades de las capas de identidad más próximas al individuo, y por prestar menos atención al área de cobertura, partiendo de las teorías de la proximidad cultural (Straubhaar, 2008). Se entiende, en definitiva, por prestadores de proximidad aquellos que desarrollan una función clave de representatividad y de información en el territorio, es decir, que emiten una programación destinada a visibilizar las informaciones del territorio más cercano, basada en la representación de las comunidades locales, y que aportan descentralización al modelo audiovisual.

\section{Referencias bibliográficas}

Almiron, N.; Narberhaus, M.; y Mauri, M. (2016). «Mapping media accountability in stateless natioins: The case of Catalonia». En: Catalan Journal of Communication \& Cultural Studies, 8:2, p. 207-225.

Anderson, B. (1983). Imagined Communities: Reflections on the Origin and Seread of Nationalism. New York: Verso.

Castells, M. (1997). La Era de la información: economía, sociedad y cultura. Vol. 2. El poder de la identidad. Madrid: Alianza.

Civil i Serra, M.; Blasco Gil, J. J.; Guimerà i Orts, J. A. (eds) (2015), Informe de la comunicació a Catalunya 2013-2014. Barcelona: Generalitat de Catalunya. Colección Lexikon Informes.

Corominas, M. (2009). Televisió local a Catalunya. Barcelona: IEC. Recuperado el 13 de febrero de 2014 de http://www.portalcomunicacion.com/ocl/download/Corominas. pdf

Corominas (2001). Radiotelevisió pública i pluralisme informatiu a Catalunya. Quaderns del CAC,(11, pp. 37-46.

Corominas, M.; Bonet, M.; Fernández Alonso, I.; Guimerà i Orts, J.A., Sanmartín, J. y Blasco Gil, J.J. (2007). «Televisión digital terrestre local (TDT-L) en España: los concesionarios privados». En: Zer, 22, pp. 69-95. 
Costera Meijer, I. (2010). «Democratizing Journalism? Realizing the citizen’s agenda for local news media». En: Journalism Studies, 11.3, pp. 327-342.

Domingo, D.; Alborch, F.; Araüna, N. (2009). La informació de proximitat als informatius televisius catalans. Bellaterra: Observatori de la Comunicació Global (InCom-UAB).

Fernández Alonso, I.; Corominas, M.; Bonet, M.; Guimerà i Orts, J.A.; Sanmartín Navarro, J. (2007). «Políticas de implantación de la TDT local en España (2005-2006): los casos de las comunidades autónomas de Islas Baleares, Madrid, Región de Murcia, Comunidad Valenciana, Galicia, Cataluña y Aragón». En: Observatorio (OBS^), 1(1), pp. 205-224.

Fernández-Quijada, D.; SellasS, T.; Bonet, M. (2013). «Media systems and stateless nations: Catalan and Welsh radio in comparative perspective». En: Trípodos, 33, p. 13-32.

Fastrez, P.; Meyer, S. (1999). Télévision locale et proximité. Recherches en communication, 11, pp. 143-167.

Fiske, J. (1987). British cultural studies and television. Londres/Nueva York: Routledge.

Guibernau, M. (2002). Naciones sin estado. Escenarios políticos diversos. En: Cuadernos de derecho judicial, 5, pp. 263-303.

Gifreu, J.; Corominas, M. (1991). Construir l'espai català de comunicació. Barcelona: Generalitat de Catalunya, Centre d'Investigació en la Comunicació.

Guimerà i Orts, J.A. (2012). «La conceptualizació de l'objecte d'estudi en la recerca sobre comunicació local a Catalunya (1981-2006): de mesocomunicació a mitjans de proximitat». En: Trípodos, 30, pp. 111-123.

Guimerà i Orts, J.A. (2010). «Polítiques de televisió, canvi tecnològic i proximitat a Catalunya». En: Quaderns del CAC, 13, pp. 13-21.

Guimerà i Orts, J.A. (2007). La televisió local a Catalunya: gestació, naixement $i$ transformacions (1976-2006). Barcelona: Consell Audiovisual de Catalunya (CAC).

Guimerà i Orts, J.A. (2006a). La televisió local a Catalunya: gestació, naixement i transformacions (1976-2005). Bellaterra: Universitat Autònoma de Barcelona. [Tesis doctoral en línea] http://www.tdx.cat/bitstream/handle/10803/4192/jago1de1.pdf?sequence=1

Guimerà i Orts, J.A. (2006b). «La televisió local a Catalunya: un model en profunda transformació». En: Quaderns del CAC, 26, pp. 141-149.

Guimerà i Orts, J.A.; Alborch, F. (2011). «La crisis de la TDT local pública en España: el caso de Cataluña». En: Revista Latina de Comunicación Social, 66, pp. 1-22.

Guimerà i Orts, J.A.; Monedero, C.d.R.; Martori, A. (2017). Digitalización, crisis económica y televisión local pública en España. Los casos de Andalucía y Cataluña (20102015). Revista latina de comunicación social, 72, 574-589.

Jankowski, N.W.; Prehn, O. (2002). Community media in the information age. Perspectives and prospects. Creskill: Hampton Press.

Kraidy, M.M. (2005). Hybridity, or the cultural logic of globalitzation. Philadelphia: Temple University Press.

La Pastina, A.C.; Straubhaar, J.D. (2005). «Multiple proximities between television genres and audiences the schism between telenovelas' global distribution and local consumption». En: Gazette, 67(3), pp. 271-288. 
López, B. (2000). «Programación y contenidos de las televisiones de proximidad en Europa». En: Vacas Aguilar, F. (2000). Televisión y desarrollo: las regiones en la era digital. Badajoz: Consejería de Educación, Ciencia y Tecnología.

Martori, A. (2016). «Televisión de proximidad en naciones sin Estado. Comparación del caso catalán y escocés». En: AdComunica, revista científica de estrategias, tendencias e innovación en comunicación, 11, pp. 43-59.

Martori, A. (2014). «Televisión pública y era digital: las emisoras de proximidad en Cataluña como caso paradigmático en su adaptación a Internet». En: Obra digital, 7, pp. 9-27.

Matterlart, A. (1998). La munidalización en la comunicación. Barcelona: Paidós.

Metzgar, E. T.; Kurpius, D. D.; Rowley, K. M. (2011). «Defining hyperlocal media: Proposing a framework for discussion». En: New media \& society, 13(5), pp. 772-787.

Monedero, R. (2012) «La televisión local pública como víctima de una desacertada evolución digital». En: Actas - IV Congreso Internacional Latina de Comunicación Social IV CILCS - Universidad de La Laguna.

Moragas, M. de (1985). «Interpretació teòrico-política de la noció de mesocomunicació». En: 1eres Jornades sobre Meso-comunicació a Catalunya, p. 15-37. Bellaterra: UAB.

Moragas, M. De; Domingo, D.; López, B. (2001). «Internet and Local communications, first experiences in Catalonia. En: Jankowski, N. (ed.) (2001). Community Media in the Information Age. Cresskill: Hampton Press.

Moragas, M. de; Garitaonandía, C.; López, B. (1999). Televisión de proximidad en Europa. Experiencias de descentralización en la era digital. Bellaterra: Universitat Autònoma de Barcelona, Servei de Publicacions.

Moragas, M. de; López, B. (2000). «Decentralizing process and "proximity television" in Europe». En: Wang, G.; Servaes, J.; Goonasekera, A. (eds.) (2000). The New Communications Landscape. Demystifying Media Globalization. Londres/Nueva York: Routledge.

Pieterse, J.N (2004). Globalization or empire?. Londres/Nueva York: Routledge.

Overdiek, A, (2016) «Fashion designers and their business partners: Juggling creativity and commerce». En: International Journal of Fashion Studies, 4:1, pp. 27-46.

Prado, E. (2004). La televisión local entre el limbo regulatorio y la esperanza digital. En: Documento de Trabajo 60/2004. Fundación alternativas. [En línea] http://www. almendron.com/politica/pdf/2005/spain/spain_2077.pdf

Prado, E.; Moragas, M. de (2002). Les televisions locals a Catalunya. De les experiències comunitàries a les estratègies de proximitat. Quaderns del CAC, número extraordinari. Barcelona: Consell de l'Audiovisual de Catalunya [En línea] http://www.mesadiversitat.cat/pfw_ files/cma/recerca/quaderns_cac/tvlocal.pdf

Prado, E.; Moragas, M. de (1991). Televisiones locales, tipología y aportaciones de la experiencia catalana. Barcelona: Col-legi de Periodistes de Catalunya.

Rushton, D. (1993). Citizen television. A local dimension to public service broadcasting. London: institute of Local Television Research Monography. 
Schlesinger, P. (2009), "Cultural and communications policy and the stateless nation». En: Catalan Journal of Communication and Cultural Studies, 1:1, pp. 9-14.

Sondergaard, H. (2003). «Television and National Identity in Denmark: The retorn of the Nation». En: Hjarvard, S. (ed.) (2003). Media in a globalized society. Copenhaguen: Unviersity of Copenhaguen Press, pp. 15-53.

Straubhaar, J.D. (2010). «Global, Hybrid or Multiple? The New Cultural Geography of Identities». En: Annual meeting of the International Communication Association. Singapore: Suntec Singapore International Convention \& Exhibition Centre. Vol. 22.

Straubhaar, J.D. (2008). «Rethinking Cultural Proximity: Multiple Television Flows for Multilayered Cultural Identities». Paper presentado en: International Communication Association, TBA, Montreal, Quebec, Canada [En línea] http://citation.allacademic. com/meta/p232732_index.html

Straubhaar, J.D. (2007). World television: From global to local. Los Angeles: Sage.

Straubhaar, J.D. (1991). «Beyond Media Imperialism: Assymetrical Interdependence and Cultural Proximity». En: Critical Studies in Mass Communication, 8, pp. 39-59.

Stiglitz, J. (2002). El malestar en la globalización. Editorial Taurus: Argentina.

Townend, J. (2015). «Hyperlocal media and the news marketplace». En: Barnett, S.; Townend, S. Media Power and Plurality: From Hyperlocal to High-Level Policy. Palgrave Macmillan, pp. 83-98.

Vila, J. (2015). «La comunicació local en l'ecosistema comunicatiu català. Informe de la Comunicació de Catalunya, 2013-2014». En: Civil, M.; Corbella, J.M.; Ferré, C.; Sabaté, J. (2015). Informe de la Comunicació de Catalunya. 2013-2014. Barcelona: Generalitat de Catalunya, Incom.

Williams, A.; Barnett, S.; Harte, D; Townend, J. (2014). The state of UK hyperlocal community news: findings from a survey of practitioners. Discussion Paper. Universitat de Cardiff [En línea] http://sro.sussex.ac.uk/67699/1/hyperlocal-community-news-in-the-uk-2014. pdf

Zallo, R. (2013). «Polítiques de comunicació a les nacions sense estat. El cas espanyol». En: Civil i Serra, M.; Blasco Gil, J.J.; Guimerà i Orts, J.A. (eds.) (2013). Informe de la comunicació a Catalunya 2011-2012. Barcelona: Generalitat de Catalunya. Col-lecció Lexikon Informes. 